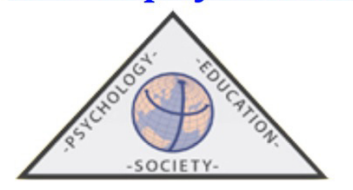

\title{
Estudio sobre la motivación en alumnado infantil de español como lengua extranjera y estrategias didácticas
}

\author{
Margarita Isabel ASENSIO PASTOR
}

Universidad de Almería

(Recibido el 15 de Marzo de 2020, Aceptado el 21 de Junio de 2020)

RESUMEN: La enseñanza-aprendizaje de español a niños no hispanoparlantes es un campo de trabajo que requiere de una especialización por parte del docente de lengua extranjera. Los procesos que intervienen en ella son un tanto más complejos que en el caso del adulto, sobre todo, porque este tipo de discente no parte de un interés motivacional inicial por lo que se requiere de un trabajo docente estimulante. El presente artículo presenta los datos de un estudio etnográfico motivacional en estudiantes de ELE a niños de entre 6 y 9 años en dos contextos distintos (Jordania e Italia). Los datos muestran un alumnado inicialmente con poco interés por estudiar español y, por tanto, sin motivación previa para ello. Será la labor del docente la que favorezca la motivación por el estudio de esta lengua. Para ello, ofrezcamos unas orientaciones didácticas y estratégicas para trabajarla en un aula de ELE infantil.

Palabras clave: español lengua extranjera (ELE); niños/niñas; motivación; estrategias didácticas; estudio etnográfico.

\section{Study on the Motivation in Children's Students of Spanish As a Foreign Language and Didactic Strategies}

\begin{abstract}
Teaching-learning Spanish for non-Spanish-speaking children is a field of work that requires specialization by the foreign language teacher. The processes involved in it are somewhat more complex than in the case of the adult, above all, because this type of learner does not start from an initial motivational interest, which requires stimulating teaching work. This paper presents the data of motivational ethnographic study in SFL students of children between 5 and 9 years old in two different contexts (Jordan and Italy). The data shows students initially with little interest in studying Spanish and, therefore, without prior motivation to do so. It will be the work of the teacher that favors motivation for the study of this language. To do this, we offer some didactic and strategic guidelines to work on in a SFL children's classroom.
\end{abstract}

Keywords: Spanish Foreign Language; Children; Motivation; Didactic Strategies; Ethnographic Study.

Correspondencia: Margarita Isabel Asensio Pastor. Ctra. de Sacramento, s/n, 04120. Almería. E-mail: masensiop@ual.es 


\section{Introducción}

La enseñanza-aprendizaje de español como lengua extranjera a niños, a partir de ahora ELEN, suele venir marcada por un hecho generalizado y es que los niños, sobre todo de corta edad, no suelen ser los que toman la decisión libre y voluntaria de estudiar esta lengua. Esto hace que sea un alumnado con desinterés inicial por el aprendizaje de español, así como una baja o nula motivación por estudiarlo como observamos en dos contextos de aprendizaje ELEN en los que trabajamos tan dispares como es Jordania e Italia.

No obstante, debemos aclarar que nos referimos a aquellos estudiantes que no tienen ninguna relación lingüística con el español (de tipo familiar o por su residencia), ni tampoco tienen ninguna vinculación afectiva por esta lengua. Desde nuestra experiencia como docentes con este contexto, observamos que la motivación en el aula ELEN resulta clave, pues hace que se produzca interés por esta lengua y, en consecuencia, que exista aprendizaje y continuidad con su estudio.

Un repaso bibliográfico revela la importancia que esta ha tenido en el terreno de la pedagogía educativa. Sin embargo, la bibliografía relativa a la enseñanza de ELE empieza a ser más profusa a principios del siglo XXI (Al-momani y Asensio Pastor, 2014; Moreno, 2014; Vilches, 2011, Marins de Andrade, 2010; Minera, 2009, entre otros). Con respecto al estudiante menor, la bibliografía es más reducida (Alcaraz, 2007; Palomino Hernández, 2012; Medina y Mora, 2017).

Así pues, el objetivo de este artículo es el de ofrecer los resultados sobre un estudio realizado sobre la motivación en el aula ELEN en los contextos mencionados al inicio de este artículo y, a partir de estos, proporcionar una reflexión sobre qué estrategias didáctica emplear en esta clase para, en definitiva, incentivar y favorecer la motivación como motor del aprendizaje entre discentes de ELEN:

"se ha escrito mucho sobre la motivación de los estudiantes (...) sin embargo, la mayoría de ese material se ha dirigido a los investigadores (con objeto de facilitar estudios posteriores) en vez de a los profesionales a fin de facilitar su labor docente". (Dörnyei, 2008: 45-46).

Para ello, comenzamos con los presupuestos epistémicos sobre la motivación en la enseñanza de lenguas extranjeras; tras ello, nos detendremos en los contextos de estudio observados y los datos obtenidos de su análisis para, a continuación, mostrar los retos y orientaciones estratégicas-didácticas para motivar en una clase de ELEN. Cerramos este artículo con las conclusiones a las que hemos llegado.

\section{Marco teórico}

\section{Aproximación al concepto "motivación"}

Comenzamos esta aproximación al concepto de motivación valiéndonos de la definición que nos proporciona el Diccionario de la Real Academia Española (en línea). Este lo define como el "conjunto de factores internos o externos que determinan en parte las acciones de una persona" (RAE, en línea). Por tanto, se trata de aquello que influye y estimula el interés de alguien para hacer algo, para aprender, etc. 
Por su parte, el Diccionario Akal de Pedagogía (2001) define el concepto "motivación" unido al de "enseñanza" y lo entiende como "la activación de la disponibilidad para el aprendizaje y la acción de los alumnos que se produce mediante la interacción de estímulos pedagógicos en la enseñanza y actitudes personales" (p. 121). De ahí que la motivación sea lo que nos mueve a hacer algo y donde su ausencia también es significativa.

Ahora bien, este término que presenta una serie de limitaciones y problemas (Madrid, 1999: 9-10, siguiendo a Rodicio, 1999). Por un lado, esta no es tangible, aunque podemos observarla a través de la conducta y la actuación del estudiante. Así, está asociado al estudio del comportamiento y también a procesos como los de necesidad, intenciones u objetivos, entre otros.

En este sentido, desde el punto de vista de la investigación, resulta complicado describir el comportamiento motivacional. Debemos hacerlo en función de la interactuación del estudiante con su entorno mediante las observaciones directas, los cuestionarios, las entrevistas o las reacciones a estímulos, por citar algunas.

Por otro lado, la motivación es un elemento más de los que determinan el proceso de aprendizaje y suele asociarse a conceptos como el de grado “el grado de motivación”. Desde esta perspectiva, es innegable que el docente puede manipular y controlar la motivación (conducta del docente, tipos de agrupamiento y actividades, etc.).

\section{La motivación y la enseñanza de ELE}

La motivación ha sido abordada desde distintos presupuestos disciplinares: el psicoanálisis freudiano, la teoría de los impulsos de Hull, del logro de Atkinson, el aprendizaje social de Rotter, la atribución de Weiner, el punto de vista humanista de Rogers o las construcciones personales de Kelly, entre otras (Madrid, 1999: 26-27).

En este sentido, la enseñanza de LE lo ha tenido en cuenta generando sus propios paradigmas de investigación sobre la motivación y el aprendizaje de lenguas extranjeras. Así pues, Arnold y Foncubierta (2019) -siguiendo a Dörnyei (2009: 16)- han considerado al menos tres fases en la evolución del estudio de la motivación con relación a la enseñanza de lenguas extranjeras:

- El período psico-social que parten de los trabajos de Gardner y Lambert (1972) hasta los años noventa y se propone una motivación instrumental y otra motivación integradora. Se parte de los modelos socioeducativos del medio en que se desenvuelven los sujetos y dependiendo de las diferencias individuales tales como la inteligencia, la aptitud para las lenguas, la motivación, las situaciones de ansiedad, de los contextos donde tienen lugar los procesos de enseñanza formal o informal se pueden explicar los resultados finales del aprendizaje.

- El período de la cognición situada durante los años noventa que procede de la psicología de la educación. Trascendiendo los conceptos de motivación extrínseca y motivación intrínseca (Deci y Ryan, 2000; Ryan y Deci, 2002). Por motivación intrínseca se entiende, aquella que está sustentada en la satisfacción inherente a la propia actividad (Ryan y Deci, 2002, p.10), mientras que la extrínseca no por el que se acometen una serie de acciones por razones instrumentales a contingencias externas.

- Nuevos enfoques desde principios del siglo XXI enfocada en los conceptos de motivación e identidad. Aquí podemos situar a Dörnyei (2009) y su sistema motivacional 
del yo o una versión más reciente llamada sistema no linear dinámico de la motivación (Dörnyei, MacIntyre y Henry, 2015)

Estos posicionamientos teóricos suelen estar centrados en dos aspectos: por un lado, los adultos y, por el otro, el contexto de inmersión lingüística de segundas lenguas. En este sentido, sigue operativa la propuesta de Maslow cuya pirámide es posible adaptar a las de necesidades comunicativas del alumno inmigrante (Asensio Pastor, 2016, p.198).

En términos generales, podemos entender la motivación como un constructo hipotético que explica los procesos mentales que instigan y sostienen la actividad dirigida a un objetivo. De ahí que la motivación sirva para explicar la acción, la intención y la toma de decisiones que una persona adopte respecto a la adquisición y el uso del nuevo código lingüístico que se estudia; si bien, en el marco ELEN hay otras cuestiones:

- La edad del estudiante porque condicionará sus posibilidades lectoescritoras, su conocimiento del mundo, sus capacidades fonadoras, etc.

- La motivación -de los estudiantes o de padres/tutores- para inscribirse en el curso.

Como suceda con otro tipo de docencia, habrá otras cuestiones que vendrán condicionadas por las anteriores como la labor docente, el material a emplear, etc.

\section{Método}

\section{Diseño de investigación}

La reflexión y orientación metodológica que aquí recogemos parte de los datos obtenidos de una investigación previa de corte cualitativo basado en la práctica etnográfica (Velasco y Díaz de Rada, 2006). La elección de este tipo de método se debió a nuestra propia involucración en el proceso didáctico.

\section{Sujetos de la investigación}

La investigación se realizó en dos entornos educativos distintos: el primero fue en el Instituto Cervantes de Amán (Jordania) y el segundo, en un colegio italiano -Convitto Nazionale Umberto I de Turín-. En ambos casos, se trata de un aprendizaje extracurricular y dentro del marco de la enseñanza de español como lengua extranjera (ELE), es decir, en un contexto exolingüe de aprendizaje del español.

El primer grupo se compuso de siete alumnos con edades comprendidas entre los 5 y los 8 años que realizaban un curso de tres horas semanales los sábados con un total de treinta. Este grupo se caracteriza, desde el punto de vista lingüístico, por una peculiar situación lingüística. Nos encontramos con estudiantes que tienen el árabe como lengua materna ${ }^{1}$.

1 Conviene aclarar que la lengua árabe está sometida a lo que se conoce con el término de diglosia, es decir, la presencia de una variedad considerada alta, culta y formal que correspondería con el árabe clásico y otra variedad propia del ámbito familiar y coloquial que correspondería a los dialectos. No obstante, todos los países árabes tienen, generalmente, más de una variedad dialectal. La existencia de varios dialectos en un mismo territorio se debe, entre otras cuestiones, a la propia migración entre las zonas, como del lugar de procedencia (urbano, del campo o beduino, principalmente). En el caso de Jordania, hay que recordar que cerca del $70 \%$ de su población tiene origen palestino, lo que queda también reflejado en la variedad dialectal de los hablantes en este país (Asensio Pastor, 2018). 
Por otro lado, todos los estudiantes de este curso tienen el inglés como lengua de instrucción en el colegio. De hecho, esta lengua también está presente en el hogar y como instrumento de sociabilización. Estamos ante alumnos bilingües. Además de lo expuesto, todos estos estudiaban francés en la escuela.

En cuanto a la segunda experiencia didáctica, esta tuvo lugar en Italia. Concretamente en un colegio que congrega desde Educación Primaria hasta el equivalente a Bachillerato español (Liceo) de Turín -Convitto Nazionale Umberto I-. Se trató de un curso extracurricular con un total de cuarenta alumnos distribuidos en dos grupos en función de la edad: uno de 6-7 años y otro grupo de 8-9 años con dos cursos (primer cuatrimestre de 20 horas y segundo cuatrimestre 20 horas; dos horas una vez a la semana).

El perfil lingüístico de estos estudiantes es menos complejo que el anterior, puesto que todos tienen como lengua materna el italiano y estudian inglés en el colegio como LE sin ningún caso de bilingüismo.

Tabla 1. Características de los grupos estudiados

\begin{tabular}{lllll}
\hline \multicolumn{2}{c}{ Instituto Cervantes } & \multicolumn{3}{c}{ Colegio italiano } \\
\hline Edad & $5-8$ años & Edad & Grupo 1 & Grupo 2 \\
Lengua materna & Árabe dialectal e inglés & Lengua materna & $6-7$ años & $8-9$ años \\
Lengua de instrucción & Inglés & Lengua de instrucción & Italiano & \\
Curso de español & 30 horas & Curso de español & $\begin{array}{l}\text { Dos grupos } \\
20 \text { horas }\end{array}$ \\
& 2 horas, 1 vez a la semana & & 2 horas, 1 vez en semana \\
Años de realización & 2006 & & $\begin{array}{l}2012 * \text { se programó para el } \\
\text { siguiente curso, pero no se } \\
\text { realizó al dejar Italia. }\end{array}$ \\
& 2007 & Años de realización & 2013 \\
\hline
\end{tabular}

\section{Instrumentos de investigación}

Las reflexiones y orientaciones didácticas parten de un estudio previo de tipo cualitativo etnográfico. Desde estos presupuestos de investigación, los instrumentos que hemos empleado para la recogida de datos en el trabajo de campo, es decir, la intervención didáctica en el aula de ELEN a través de la observación participante, el diario de campo y las entrevistas. En este sentido, Woods (1987: 135) señala que "cuando se observa, se entrevista, se toman notas de campo y se confecciona el diario de investigación, la labor del etnógrafo no se limita a "registrar". También hay en ello reflexión". Además, el observador participa “abiertamente o de manera encubierta, en la vida diaria de las personas durante un periodo de tiempo, observando qué sucede, escuchando qué se dice, haciendo preguntas; de hecho, haciendo acopio de cualquier dato disponible que pueda arrojar un poco de luz sobre el tema en que se centra la investigación" (Hammersley y Atkinson, 2005: 15), para reflexionar durante el propio proceso y posteriormente, ofreciendo los datos obtenidos del análisis final. 
Con respecto a las entrevistas, fueron semiestructuradas y dirigidas tanto a los alumnos como a las personas a su cargo. Estas fueron grabadas en formato audio.

En el caso de los niños y las niñas, se realizaron de dos maneras: la primera de manera conjunta, los últimos diez minutos de la clase con preguntas del tipo ¿qué es lo que más os ha gustado hacer hoy? o con preguntas directas ¿os ha gustado cantar?; también se les pasó un breve cuestionario al final del curso para que marcaran con una $\mathrm{x}$ las actividades que más le gustaron. Asimismo, se realizaron breves entrevistas no superiores a cinco minutos (recurriendo a su propia lengua); entre las cuestiones destacamos lo que más les ha gustado de las clases, si les gustaría continuar estudiando y los motivos.

En el caso de los adultos, primero se hicieron entrevistas informales a la hora de recoger a los niños/las niñas; al final del curso, se les pidió una formal con una duración máxima de diez minutos en los que se les preguntó, por un lado, el motivo de matricular a su hijo/hija en el curso de español y, por otro, si había observado interés y motivación de estos por la lengua; no obstante, al ser una entrevista semiestructurada, esta fue variando según el progenitor y sus respuestas. En total, disponemos de 10,25 horas de grabaciones.

\section{Datos}

\section{La motivación para estudiar español}

Como ya expusimos al inicio de este artículo, el estudio del español por parte de estos estudiantes no nace de ninguna necesidad en los términos que Maslow propuso y que suele aplicarse a la enseñanza de segundas lenguas (Asensio pastor, 2016). La decisión de estudiar español fue tomada por los adultos responsables de estos niños (padres/tutores legales) y los motivos para ellos son:

- En el caso del primer grupo, que los niños dispusieran de una tercera o, incluso, una cuarta LE; solo uno de estos lo hacía porque el padre se encontraba trabajando en España y, al final de año, se trasladaría toda la familia allí.

- Con respecto al segundo grupo, tampoco existe una motivación previa de estos por el español, excepto dos de ellos con hermanos mayores que se encontraban estudiando en el Liceo esta lengua, en la Sección Español, es decir, un centro adscrito a la acción educativa española en el exterior. ${ }^{2}$ No obstante, cabe la posibilidad de que estos estudiantes continúen sus estudios en este Liceo.

De lo que se desprende de estos datos es que no existe una motivación intrínseca al estudiante. Por tanto, esto se convierte en un reto para el/la docente para que el primer día quisieran asistir y disfrutar de las clases, así como por aprender español y mantener la motivación hasta el final del curso.

2 Ver información relativa a las Secciones Españolas de Educación en el Exterior: https://www.educacion.gob.es/centros/buscar.do?shortcut $=1 \&$ codaut $=60 \&$ nombreaut $=$ EXTRANJERO\&codprov $=00 \&$ combopais $=380 \&$ textopais $=$ ITALIA\&ssel_natur=0 bibliografía . La importancia del español en este tipo de liceos internacionales españoles es que implica realizar la maturità en español (el equivalente al examen final de Bachillerato para poder acceder a los estudios universitarios). 
Por otro lado, ambos cursos de español son extraescolares y extracurriculares. A lo que debe sumarse el hecho de no ser la única actividad fuera del horario escolar de estos estudiantes. Desde esta perspectiva, nos encontramos con estas cuestiones:

- Los alumnos asistentes tienen una sobrecarga de actividades por lo que el interés por, en este caso el español, decrece debido al cansancio tanto físico como mental. Además, en el caso del primer grupo, estos estaban sometidos a un alto grado de exposición a otras lenguas.

- Relacionado con la anterior, se trata de alumnos que respondieron mejor a actividades diseñadas desde una perspectiva lúdica, especialmente, con movimiento y música. Al final del curso, todos se sabían las canciones empleadas en clase o, al menos, varias de estas, así como nombrar cosas/colores/animales, etc. en español.

- En cuanto a las entrevistas a los adultos (responsables de los niños/las niñas), el 87,7\% afirma que sus hijos/hijas les cantan las canciones. De igual modo, todos los entrevistados, a excepción de tres de ellos, manifestaron si intención de volver a matricular a sus hijos/hijas en las clases de español al siguiente año, si se volviera a repetir el curso. Las excepciones están relacionadas con el cambio de residencia de país en el caso de un alumno jordano y los otros dos italianos no indicaron el motivo.

Además, la docencia con este tipo de alumnado está determinada por una serie de limitaciones propias de su edad cognitivas y psicomotrices, conocimiento del mundo (experiencia), desarrollo de la lectoescritura y el grado de competencia lecto-escrita de estos, entre las principales cuestiones.

En otro orden de cuestiones, una mención concreta merece el material que se empleó en la docencia de cada uno de estos cursos. En el primer caso, nos encontramos con mayores dificultades, sobre todo, para la compra del mismo; en este sentido, recurrimos al material del que dispone este centro, aunque estaba poco actualizado. Esto hizo que se tuviera que recurrir a la creación de nuestro propio material. Como resultado de ello, se realizó una programación de curso de español a niños no hispanoparlantes que se encuentra colgado en la intranet del Instituto Cervantes.

Con respecto al segundo grupo, hemos de destacar que la presencia de manual estuvo motivada por el equipo directivo y los familiares. Para ello, se optó por Colega de la editorial Edelsa por adaptarse al nivel cognitivo, psicomotriz y cultural del alumno con actividades que tuvieran en cuenta otras destrezas propias de su edad (pintar, dibujar, recortar, pegar...), con música y juegos; también se recurrió a la elaboración o a la adaptación de actividades.

Cada una de estas modalidades, evidencian unas ventajas e inconvenientes. En el primer caso, se trata de la creación de un curso ad hoc realmente centrado en el alumnado. Sin embargo, es un material que se va realizando a lo largo del curso, aunque se partiera de una programación inicial. Requiere de mayor implicación creativa por parte del docente y de tiempo. Por el otro lado, el manual nos ofrece ya una programación y cierta sistematización de las unidades y actividades que, en ocasiones, requería también de nuestra implicación creativa en tanto que algunas actividades se quedaban cortas, necesitaban ser ampliadas, adaptadas o reconducidas, según las necesidades del alumnado. 
Además de lo expuesto, ambas experiencias revelaron la necesidad de realizar actividades lúdicas en las que se empleara todo el cuerpo: bailar, movimiento en general o canto se muestran como las más eficientes y eficaces a la hora de crear un clima motivador. Estas ayudan a concentrarse y, en definitiva, a aprender divirtiéndose sin sentir el peso del proceso de aprendizaje de una lengua como en un centro escolar.

\section{Estrategias y orientaciones didácticas}

En los apartados que siguen, ofrecemos una serie de reflexiones didácticas para hacer la enseñanza-aprendizaje de ELEN de manera motivacional. Para ello, abordaremos la cuestión atendiendo al siguiente orden expositivo: en primer lugar, atenderemos a los factores internos del discente en relación con el proceso de enseñanza-aprendizaje de ELE y, posteriormente, nos centraremos en los factores externos a dicho proceso.

\section{Factores internos al discente}

Toda persona dispone de una serie de aptitudes intrínsecas a sí misma que no dependen de nosotros y que determinan nuestras capacidades para -en el caso que nos ocupa- aprender una lengua distinta a la materna. Además, en el caso del alumnado infantil en un aula de LE, hemos de tener presente el momento psicofísico y madurativo de este. Aspectos, por otra parte, que lo diferencian del discente adulto y que, en consecuencia, afectarán a la enseñanza de LE.

Así pues, hemos establecido la siguiente clasificación teniendo como base principal la propuesta de Brooks (1964) y Slobin (1973):

- Entre los 2 y los 4 años, el niño es capaz de combinar una o más palabras -que van de lo general a lo preciso-. Rodríguez y Valera (2004: 165) advierten de que es un momento en el que el niño está en continuo movimiento y comienza a emplear estrategias de sociabilización con el resto de compañeros.

- A partir de los 4 hasta aproximadamente los 7 años, es capaz de estructurar el conocimiento, dispone de capacidad de síntesis, construye oraciones, generaliza y reflexiona metalingüística con tendencia a los seis años (Slobin, 1973) de crear una forma propia de comunicar sus deseos y pensamientos. También dispone de mayor control corporal, sobre todo, de la musculatura de la muñeca y las manos, es decir, comienza a disponer de psicomotricidad fina que le permitirá comenzar con el proceso de escritura y comienza a tener, progresivamente, capacidades de sociabilización y empatía (Sadurní et al., 2008: 177-178).

- Desde los 7 u 8 años hasta los 10 años, los niños experimentan con la lengua desde el punto de vista sintáctico y comienzan a disponer de un lenguaje propio que se manifiesta, sobre todo, a los 14 o 15 años, con un empleo más elaborado del mismo en el que aparece lo metafórico y la capacidad de abstracción. Por otro lado, sufren lentos procesos de desarrollo físico con respecto a etapas anteriores, pero que resultan más llamativos con efectos en el desarrollo social y de su personalidad que pueden también incidir en su autoimagen (Sadurní et al., 2008: 198).

El niño/la niña, a diferencia del adulto, cuanta menor edad tiene, menos definido está su tipo de inteligencia (Gardner, 2003), carece de menos estrategias de estudio y dominio de la 
lectoescritora y dispone de un conocimiento limitado cultural, así como otros aspectos (Asensio Pastor, 2016):

- Desde un punto de vista de dificultad, encontramos dos cuestiones: a menor edad, mayor tendencia a ser absorbentes y de llamar la atención del adulto, así como un bajo índice de concentración.

- Por otro lado, desde un punto de vista facilitador del proceso de aprendizaje se caracterizan, en términos generales, por no tener miedo a equivocarse; ser competitivos por lo que intentarán ser los primeros en resolver los ejercicios; les gusta relacionarse con sus compañeros; les gusta hablar de sí mismo y de su entorno.

\section{Factores externos al discente}

El docente debe colaborar para que las propuestas de intervención en el aula de ELEN, por lo expuesto, resulten motivacionales por lo que los objetivos que nos proponemos para un curso de estas características, parte de estos dos aspectos:

- La creación de un clima inicial motivacional

- El mantenimiento la motivación del alumno durante el tiempo que dure el curso

Para la consecución de este ambiente, las siguientes variables resultan decisivas:

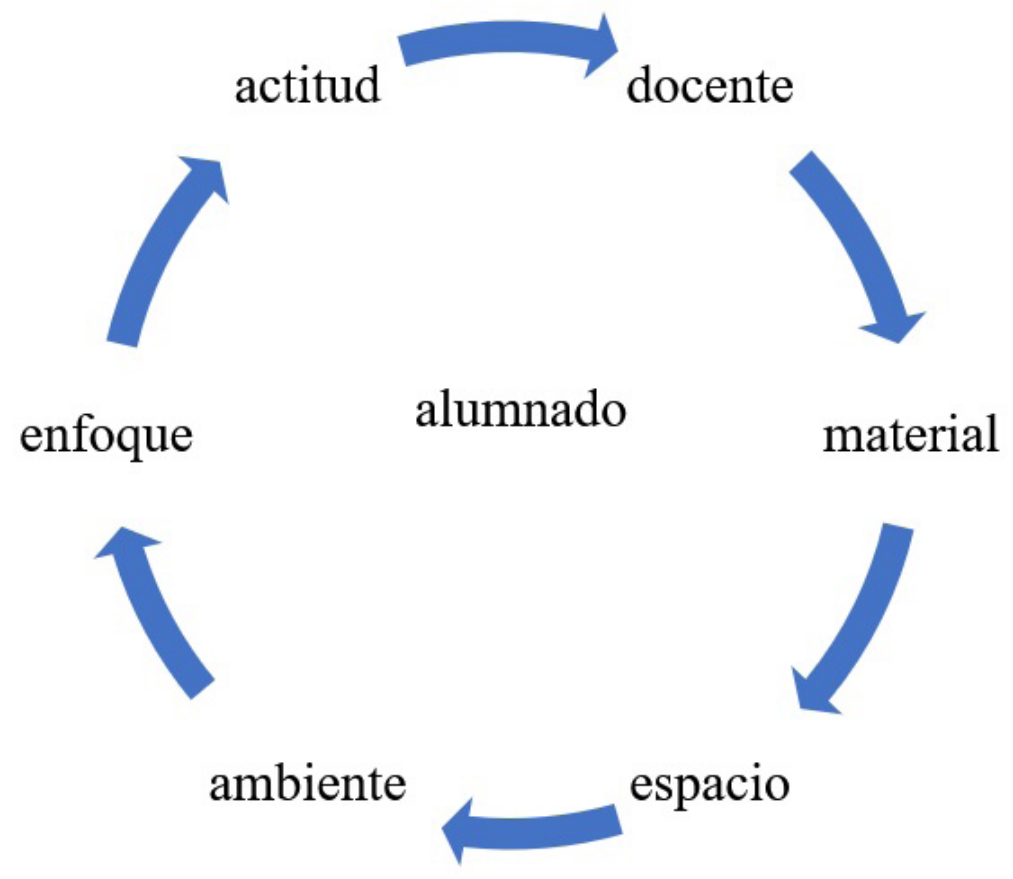

Gráfico 1. Proceso de enseñanza-aprendizaje.

Fuente: elaboración propia.

Con el gráfico anterior, queremos hacer hincapié en que el estudiante es el centro del proceso de enseñanza-aprendizaje y que todos los elementos mencionados anteriormente han de tenerse presentes de forma holística e interrelacionados para que la enseñanza sea un proceso y no un producto. 


\section{El rol del docente}

El docente tiene un papel relevante en este tipo de enseñanza, pues la eficacia de este incide en el rendimiento del alumnado (Ashton, 1985). Este ha de ser proactivo y motivado para favorecer un ambiente positivo docente-discente y entre discentes, evitando la ansiedad y eliminado la idea negativa del error. También debe guiar, controlar y marcar los tiempos dentro del aula. De acuerdo con Prabhu (1999: 169), la eficacia de la labor docente no solo radica en el método que este utilice, sino que esta dependerá en gran medida de aspectos tales como lo activo, motivador y operativo que sea este para involucrar en el proceso de enseñanza al discente. Por tanto, porponemos las siguientes estrategias de intervención didáctica en el contexto de ELEN para las cuales hemos seguido y adaptado las de Wlodkowski (1986: 42 cit. en Dörnyer, 2008: 49):

- Claridad expositiva de los contenidos y adaptados al nivel de competencia comunicativa de los mismos. Así como, en relación a las capacidades cognitivas de los estudiantes, sus ritmos de aprendizaje y las inteligencias (Gardner, 1985).

- Emplear ejemplos que sustenten las explicaciones y los ejercicios que deban realizar los estudiantes.

- Describir correctamente qué y cómo deben hacerse las actividades y que se hagan en el aula mandando el mínimo fuera de esta; aquello que sea para casa, que esté explicado y con muestras realizadas en clase.

- Variedad de actividades y conceder el tiempo necesario para hacerlas bajo nuestra supervisión.

- Implicar a los padres/tutores en el proceso de enseñanza-aprendizaje de los alumnos con reuniones tanto al inicio como durante el curso.

- El tratamiento del error en el aula debe cobrar una nueva dimensión positivista, es decir, que el docente no sancione, sino que vea la información que ofrece la interlengua, como señala Fernández López (1995).

- La actitud de los docentes que Martín y Zuheros (2016: 46) denominan "buenas prácticas del profesor" como son la empatía, la retroalimentación sobre las actividades que realice el alumno, así como un refuerzo positivo que ponga en valor siempre lo bueno, aunque no hemos de dejar de corregirles. Estos elementos colaborarán en desarrollar en el discente una aceptación del docente y reforzará la autoimagen y autoestima de los niños/las niñas.

\section{El enfoque, la programación, el material y el espacio}

En este apartado nos vamos a ocupar de otros aspectos que favorecen una docencia motivacional como son la elección del enfoque y método, la programación que realizamos, el material que se emplee e, incluso, el espacio y la distribución de este.

- El enfoque y metodología consideramos que deben responder a los principios comunicativos, pero también al enfoque lúdico. Hemos de conseguir que la clase sea toda una experiencia de aprendizaje y, de igual modo, divertida, a la que quieran volver. 
- La programación debe estar adaptada al nivel cognitivo y psicofísico de los estudiantes, así como un material que tenga esto en cuenta. En este sentido, la primera sesión con el alumnado es decisiva. Esta le servirá al alumnado a conocer cómo será el funcionamiento de las clases, a la propia lengua y su cultura e, incluso, al docente, entre otras cuestiones.

- Optar por realizar actividades dinámicas, variadas, creativas, imaginativas y lúdicas que involucren no solo al niño/la niña, sino a todo el grupo y, además, que se empleé todo el cuerpo (bailar, cantar, movimiento en general) que sirva para disociar la clase de español de materias escolares en las que impera la idea de estudio-esfuerzo-nota.

- El juego será fundamental porque puede ayudar a focalizar la energía del niño de forma productiva. Este estimula al discente cognitiva y afectivamente, verbal y psicomotrizmente y también social y lingüísticamente al tener que emplear la lengua para jugar (Instituto Cervantes, 2006; Consejo de Europa, 2002). Proponemos dos tipos de juegos, libres o imaginativos y regulados o sistemáticos (Labrador, 2008: 75), variados para no incurrir en el aburrimiento y la repetición, que dispongan de un nombre para que los niños los reconozcan, controlados temporalmente y con música. En estos, el docente debe ser activo, pues su falta de participación puede ser interpretada como una actividad aburrida (Asensio Pastor, 2016), aunque nuestra presencia dependerá del objetivo que queramos conseguir.

- La elección del manual y el material es esencial. La oferta editorial actual de ELEN es amplia y variada ${ }^{3}$. Sin embargo, esta no elimina la parte de creación de material específico ${ }^{4}$.

- El empleo de la tecnología de la información y la comunicación (TIC) debe incluirse en el aula porque forma parte de la realidad del alumnado infantil y puede colaborar tanto para la realización de actividades de consolidación de contenidos, como para fomentar su aprendizaje autónomo fuera del aula.

- La propuesta de actividades que se haga, además de atender a las capacidades cognitivas, psicomotrices, de conocimiento del mundo y cultural del alumnado, también deben trabajar otras cuestiones relacionadas con el aprendizaje autónomo, los valores, la sociabilización y la cooperación a modo de aprendizajes transversales que vayan más allá del propio curso de español LE que, en consecuencia, les sirva para su propio crecimiento personal.

- El espacio-aula es un microsistema (Ames y Ames, 1985) que afecta a la docencia y a la motivación. Este debe ser flexible en donde poder moverse y con un mobiliario movible

3 En estos últimos años, las propuestas didácticas de los libros de ELE infantiles prestan especial atención a la motivación. En un estudio en proceso de realización por Asensio Pastor, se observa cómo los manuales de los años 90 de español LE estaban más cercanos a los libros de adultos (temas, tipo de actividades, etc.), frente a los manuales a partir del 2000 que tienden a incluir otras actividades en las que se emplea la canción, el juego u, otras que implican cortar, pegar, dibujar o colorear; además de una tendencia a privilegiar la oralidad entre los alumnos de más corta edad para, progresivamente, ir aumentando el grado de complejidad de las actividades.

4 Con respecto a nuestra propia experiencia ELE, como vimos en lo expuesto sobre los datos de los contextos estudiados, uno por necesidad ante la ausencia de manual y otro por las circunstancias de ampliación, complementación o, incluso, redireccionación de las actividades, hace que la creatividad esté presente. En este sentido, su principal ventaja es adaptar los materiales a los discentes y no viceversa. 
que podamos adaptar a las necesidades de las actividades, así como trabajar en el suelo, sobre todo, sentados en círculo para poder hablar todos cara a cara. El aula debe estar decorada, no solo por carteles que los docentes coloquemos como estímulos visuales -sin caer en la saturación-, sino con el propio material que haga el alumno: dibujos, trabajos, etc.

\section{Conclusiones}

La motivación es una poderosa herramienta que sirve, incluso, para suplir ciertas carencias que presente los alumnos. Desde el punto de vista de la enseñanza del español como LE servirá como un instrumento capaz de hacer que los alumnos se interesen por esa nueva lengua teniendo en cuenta que no presentan una necesidad real de uso, como sí sucederá en los contextos de L2.

El docente, en el proceso de motivación de los alumnos-niños, juega un papel decisivo. Este no solo debe conocer los factores internos relativos al discente, sino que ha de controlar otros aspectos tales como la preparación del curso y su ejecución. Además, la elección del material y el enfoque de las actividades, así como la retroalimentación positiva que este haga serán determinantes para crear un clima idóneo de enseñanza-aprendizaje y redundará positiva y eficazmente en el proceso del discente. También existen otros aspectos de la docencia que son determinantes para motivar al alumnado como es el enfoque, el tipo de actividades que se realicen o el propio espacio en el que se desarrolle la docencia que deben ser tenidos en cuenta por el docente.

En definitiva, el aprendizaje de una LE a edad temprana estará fuertemente marcada por el elemento afectivo y determinarán el grado de motivación que este tipo de discente tenga con respecto a la nueva lengua a lo largo del proceso de enseñanza.

\section{Referentes}

Ames, C. y Ames, R. (1985). Research on Motivation in Education. Vol. 2: The classrom milieu. Nueva York: Academic Press, Harcourt Brace Jovanich.

Arnold, J. (2000). La dimensión afectiva en el aprendizaje de idiomas. Madrid: CUP.

Arnold, J. y Foncubierta, J. M. (2019). La atención a los factores afectivos en la enseñanza de ELE. Madrid: Editorial Edinumen.

Alcaraz Andreu, C. (2007). Motivación y edad: dos factores clave en el aprendizaje de la expresión oral. En S. Pastor Cesteros y S. Roca Marín XVIII Congreso Internacional de la ASELE La evaluación en el aprendizaje y la enseñanza del Español como Lengua Extranjera / Segunda Lengua (pp. 205-216). Alicante: Universidad de Alicante. Recuperado de https:// cvc.cervantes.es/ensenanza/biblioteca_ele/asele/pdf/17/17_0205.pdf

Al-momani, R. y Asensio Pastor, M. I. (2014). El privilegio de la afectividad en la adquisición del español LE: el caso de Jordania. En Y. Moirmoto , M. V. Pavón y R. Santamaría (Eds.) La enseñanza de ELE centrada en el alumno Recuperado de https://cvc.cervantes.es/ ensenanza/biblioteca_ele/asele/pdf/25/25_0105.pdf

Asensio Pastor, M. I. (2016). Enseñar español como lengua extranjera a niños: aportaciones teórico-prácticas.Lenguajey Textos, 44, 95-105.DOI:https://doi.org/10.4995/lyt.2016.6983. 
Asensio Pastor, Margarita I. (2018). Enseñar español como Segunda Lengua a alumnos de Educación Infantil: Retos y orientaciones metodológicas. Doblele. Revista de lengua y literatura 4: 108-21. DOI: https://doi.org/10.5565/rev/doblele.43

Asensio Pastor, M. I. (2018). La realización del fonema/q/ en las variedades dialectales del árabe de Jordania: análisis de cuentos de tradición oral. Estudios de fonética experimental. Vol. 27, 125-150. Recuperado de https://www.ub.edu/journalofexperimentalphonetics/pdfarticles/XXVII-09-MAsensio-separata_new.pdf

Ashton, P. (1985). Motivation and the Teacher's Sense of Efficacy. En C. Ames y Ames, R. Research on Motivation in Education. Vol. 2: The classroom milieu. Nueva York: Academic Press, Harcourt Brace Jovanich. 141/167.

Consejo de Europa. 2002. Marco común europeo de referencia para las lenguas: aprendizaje, enseñanza, evaluación. Madrid: Instituto Cervantes-Ministerio de Educación Cultura y Deporte: Anaya.

Deci, E.Ly Ryan, R.M. (2000) "Self- determination theory and the facilitation of intrinsic motivation, social development and well-being". American Psychologits. (pp. 6878). En http://www. vingus.com/course\%20work\%20data\%20files/MBA\%208165/self\%20determination.pdf

Dörnyei, Z. 2008. Estrategias de motivación en el aula de lenguas. Barcelona: Editorial UOC.

Fernández López, S. (1995). Errores e interlengua en el aprendizaje del español como lengua extranjera. Didáctica, 7. 203-216.

Gardner, H. (2003). La inteligencia reformulada: Las inteligencias múltiples en el siglo XXI. Barcelona: Paidós.

Gardner, R. C. (1985). Social Psycology and Second Language Learning. The Roles of Attitudes and motivation. Londres: Edward Arnold.

Gardner, R. C. y Lambert, W. E. (1972). Attitudes and motivation in second-language learning. Rowley: Newbury House.Instituto Cervantes. 2006. Plan Curricular del Instituto Cervantes. Niveles de Referencia para el español. Madrid: Instituto Cervantes, Biblioteca Nueva.

Hammersley, M. y Atkinson, P. (2005). Etnografía. Métodos de investigación. Barcelona: Paidós. Instituto Cervantes (2006). Plan Curricular del Instituto Cervantes. Recuperado de http://cvc. cervantes.es/Ensenanza/biblioteca_ele/plan_curricular/default.htm Jefatura

Madrid Fernández, D. (1999). La investigación de los factores motivacionales en el aula de idiomas. Granada: Universidad de Granada.

Marins de Andrade, P. R. (2010). Estrategias de aprendizaje y desarrollo de la motivación: un estudio empírico con estudiantes de E/LE brasileños Porta Linguarum, 14 pp. 141-160. Recuperado de https://cutt.ly/ZtgMte0

Minera Reyna, L. E. (2009). El papel de la motivación y las actitudes en el aprendizaje de ELE en un contexto de enseñanza formal para adultos alemanes. Revista Nebrija de Lingüistica Aplicada a la Enseñanza de las Lenguas, 6. Recuperado de http://www.nebrija.com/ revista-linguistica/el-papel-de-la-motivacion-y-las-actitudes-en-el-aprendizaje-de-ele-enun-contexto-de-ense $\% \mathrm{C} 3 \% \mathrm{~B} 1$ anza-formal-para-adultos-alemanes

Moreno Bruna, A. M. (2014). La motivación en el aprendizaje de E/LE de los estudiantes de traducción e interpretación en Flandes según el sistema motivacional del yo de Zoltán Dörnyei análisis cuantitativo por sondeo de la influencia de las percepciones culturales acerca de España y Latinoamérica en el proceso de aprendizaje. MarcoELE, 18. Recuperado de http://marcoele.com/descargas/18/moreno-motivacion.pdf 
Prabhu, N. S. (1990). “There is no best method-Why?” TESOL Quarterly, 24. 180-202.

Ryan, R. M. y Deci, E. L. (2000). "Intrinsic and Extrinsic Motivations: Classic Definitions and New Directions" Contemporary Educational Psychology, 25. 54-67 doi:10.1006/ ceps.1999.1020

Sadurní i Brugué, M. (coord..); Roslán Sánchez, C. y Serrat Sellabona, E. (2008). El desarrollo de los niños, paso a paso. Barcelona: Editorial UOC.

Schaub, H, y Zenke, K. G. (2001). s.v. Motivación. En Diccionario Akal de Pedagogía. Madrid: Akal. P. 121

Slobin, D. I. (1973). The ontogenesis of Language. Nueva York: Academic Press.

Velasco, H. y Díaz de Rada, A. (2006). La lógica de la investigación etnográfica. Un modelo de trabajo para etnógrafos de escuela. Madrid: Trotta.

Woods, P. (1987). La escuela por dentro. La etnografía en la investigación educativa. Barcelona: Paidós-MEC.

Vilches Furió, M. (2011). El desafío de motivar a los alumnos de ELE Análisis comparativo de dos manuales de enseñanza de ELE en torno a estrategias de aprendizaje de lengua, destrezas de lengua y motivación. https:/gupea.ub.gu.se/bitstream/2077/26151/1/ gupea_2077_26151_1.pdf 